\title{
A toolbox and procedural notes for characterizing novel zinc finger nucleases for genome editing in plant cells
}

\author{
Andriy Tovkach, Vardit Zeevi and Tzvi Tzfira* \\ Department of Molecular, Cellular and Developmental Biology, The University of Michigan, Ann Arbor, MI 48109, USA
}

Received 1 August 2008; revised 26 September 2008; accepted 9 October 2008; published online 24 November 2008.

*For correspondence (fax +734 647 0884; e-mail ttzfira@umich.edu).

\begin{abstract}
Summary
The induction of double-strand breaks (DSBs) in plant genomes can lead to increased homologous recombination or site-specific mutagenesis at the repair site. This phenomenon has the potential for use in gene targeting applications in plant cells upon the induction of site-specific genomic DSBs using zinc finger nucleases (ZFNs). Zinc finger nucleases are artificial restriction enzymes, custom-designed to cleave a specific DNA sequence. The tools and methods for ZFN assembly and validation could potentially boost their application for plant gene targeting. Here we report on the design of biochemical and in planta methods for the analysis of newly designed ZFNs. Cloning begins with de novo assembly of the DNA-binding regions of new ZFNs from overlapping oligonucleotides containing modified helices responsible for DNA-triplet recognition, and the fusion of the DNA-binding domain with a Fokl endonuclease domain in a dedicated plant expression cassette. Following the transfer of fully assembled ZFNs into Escherichia coli expression vectors, bacterial lysates were found to be most suitable for in vitro digestion analysis of palindromic target sequences. A set of three in planta activity assays was also developed to confirm the nucleic acid digestion activity of ZFNs in plant cells. The assays are based on the reconstruction of GUS expression following transient or stable delivery of a mutated uidA and ZFN-expressing cassettes into target plants cells. Our tools and assays offer cloning flexibility and simple assembly of tested ZFNs and their corresponding target sites into Agrobacterium tumefaciens binary plasmids, allowing efficient implementation of ZFN-validation assays in planta.
\end{abstract}

Keywords: zinc finger nucleases, gene targeting, tools, non-homologous end joining.

Introduction

The targeting and modification of native genomic sequences (gene targeting, GT) in yeast and animal cells is often made possible by homologous recombination (HR) between the donor and chromosomal DNA (Jasin et al., 1996). Several attempts have been made, with only little reported success, to introduce efficient HR-based GT methods for use in plant species. These include, for example, using a strong positiveand negative-selection scheme (Terada et al., 2002) or overexpressing a HR-related yeast protein in Arabidopsis plants (Shaked et al., 2005). It seems that the dominance of non-homologous end joining (NHEJ) over HR in plant DNA repair, and by implication the integration of foreign DNA into the plant genome, does not permit efficient HR between donor and chromosomal DNA (Britt and May, 2003; Siebert and Puchta, 2002; Tzfira et al., 2004). Methods are therefore needed to increase the rate of HR or interfere with the random integration process of foreign DNA in plants.

Enhanced HR can potentially be achieved in plant cells by creating genomic DSBs at the target site (Puchta, 2002). Thus, for example, expression of I-Scel, a rare-cutting restriction enzyme, has been shown to lead to a significant increase in HR-mediated GT in tobacco plants (Puchta et al., 1993, 1996), and expression of HO endonuclease has been shown to enhance intrachromosomal recombination in Arabidopsis plants (Chiurazzi et al., 1996). Expression of rare-cutters can also lead to site-specific integration of foreign DNA molecules and to induction of site-specific mutagenesis. For example, expression of I-Scel and I-Ceul 
led to deletions, insertions and targeted integration of foreign DNA molecules in the break sites of tobacco plants (Chilton and Que, 2003; Salomon and Puchta, 1998; Tzfira et al., 2003). The potential of using rare-cutters to increase the HR rate or as site-specific mutagens is thus very clear (Carroll, 2004; Paques and Duchateau, 2007). Nevertheless, their use is limited to rarely occurring natural recognition sites or to artificial target loci. Furthermore, the ability to re-engineer rare-cutters for novel DNA-target specificities is both difficult and tedious (Paques and Duchateau, 2007), limiting their use for GT experiments. One possible route to overcoming these technological challenges is the use of zinc finger nucleases (ZFNs) - synthetic restriction enzymes which can be specifically designed to cleave virtually any long stretch of double-stranded DNA sequence (for recent reviews see Carroll et al., 2006; Durai et al., 2005; Porteus and Carroll, 2005). Indeed, expression of ZFNs in living cells has been shown to produce genomic DSBs, leading to enhanced HR, site-specific mutagenesis and targeted gene addition in various species (e.g. Beumer et al., 2006; Bibikova et al., 2001; Moehle et al., 2007; Porteus and Baltimore, 2003; Santiago et al., 2008; Urnov et al., 2005). Two independent reports have demonstrated the use of ZFNs for genome modifications in plant cells. In one, ZFNs were used to increase the frequency of HR in tobacco plants (Wright et al., 2005) and in the other, ZFNs were used to induce sitespecific mutagenesis in Arabidopsis plants (Lloyd et al., 2005).

The rules and protocols for the assembly of new ZFNs have been the subject of several excellent recent reports (e.g. Carroll et al., 2006; Mani et al., 2005; Wright et al., 2006), and various tools and vectors for the design and assembly of novel ZFNs are available from Carlos Barbas's laboratory (http://www.scripps.edu/mb/barbas/zfdesign/ zfdesignhome.php) and the Zinc Finger (ZF) Consortium (http://www.zincfingers.org). It should be noted, however, that while we can potentially design ZFNs to target virtually any genomic sequence, caution needs to be exercised in their construction based solely on computational methods and their subsequent use for targeting experiments in plant cells (and any other species for that matter). Ramirez et al. (2008), for example, recently reported an unexpected failure rate for the modular assembly of new engineered zinc finger domains and pointed to some of the experimental obstacles that can hinder the assembly and evaluation of novel ZFNs. Thus, the application of ZFN technology for GT experiments requires not only the design and assembly of novel ZFNs, but also proper validation of their activity in living cells. More specifically, it is important to verify not only that the newly designed ZFNs can bind and digest in vitro target sequences, but that they can digest target sequences in vivo as well (Cathomen et al., 2008; Porteus, 2008).

Realizing the importance of analyzing and testing the binding and digestion activities of new ZFNs, several groups have developed assays for their analysis. These include, for example, a bacterial cell-based reporter assay that analyzes the DNA-binding activities of zinc finger domains (Wright et al., 2006), a cell culture-based system suitable for analyzing the digestive activity of newly designed ZFNs in mammalian cells (Porteus, 2008) and a digestion assay for in vitro analysis of ZFN digestion activity (Mani et al., 2005). While these assays can facilitate the assembly and analysis of ZFNs for in vitro use and for GT in mammalian cells and other model organisms (e.g. Carroll et al., 2008; Porteus, 2008; Zeevi et al., 2008), no specialized vectors, tools or protocols have been described to assist with the development and validation of ZFNs in plant species.

Here we describe a set of assays and vectors suitable for the assembly, expression and functional analysis of novel ZFNs in plant cells. In addition to an in vitro digestion assay, we designed three in planta digestion assays which can be used for functional analysis of newly assembled ZFNs on transiently delivered and chromosomally integrated target DNA molecules. The flexibility of our vector design, which allows easy assembly and transfer of ZFN coding sequences between bacterial and plant expression vectors, the ability to clone ZFN expression cassettes onto Agrobacterium tumefaciens binary vectors and the simplicity of our in planta digestion assays which are based on reconstruction of the GUS reporter gene in living cells, provide the user with a useful collection of plasmids and assays for the development of ZFNs for plant research and biotechnology.

\section{Results}

\section{Outline of ZFN functional assays and vector systems}

To facilitate the analysis of newly assembled ZFNs, we designed a battery of assays allowing functional analysis of the activity of ZFN monomers in vitro and in planta. Figure 1 outlines the set of assays for ZFN activity and illustrates the main vector components required for each assay. Each assay is composed of a ZFN expression cassette and a target sequence on which the ZFN activity is to be tested. Since each assay was designed for the analysis of ZFN activity under a different setting, we decided to develop the different ZFN expression plasmids with maximal flexibility, allowing the easy and simple transfer of ZFN components between the various vectors. Figure 2 illustrates the structures and generic names of the ZFN expression vectors, their derivatives and other components of our ZFN assembly and functional assays. In line with other reports describing the development of ZFNs for animal and human cell applications (e.g. Cathomen et al., 2008; Porteus, 2008), we propose that custom-designed ZFNs be assayed not just for their in vitro digestion activity but also for their ability to target stably integrated genomic sequences, before considering 

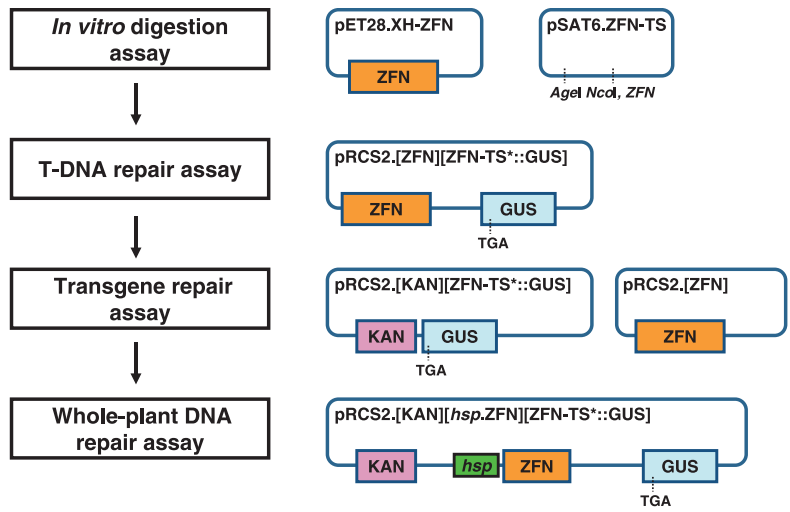

Figure 1. Outline of zinc finger nuclease (ZFN) functional assays and their vector systems.

The step-by-step comprehensive analysis of novel ZFNs is composed of four distinct assays (left panel), each based on monitoring ZFN activity using a defined set of vectors (right panel). The in vitro digestion assay tests the digestion activity of an Escherichia coli-expressed ZFN on its recognition site cloned on a target vector. The T-DNA repair assay requires the assembly of a dual-expression cassette on an Agrobacterium tumefaciens binary vector and tests the ability of a constitutively expressed ZFN to digest and repair a mutated GUS reporter gene cloned on the T-DNA region of that vector. The transgene repair assay calls for separating the ZFN expression cassette from its target site based on the activation of a mutated GUS reporter gene in transgenic calli, while the whole-plant repair assay activates the mutated GUS reporter gene in seedling or mature plant tissues upon specific activation of the ZFN. TS, ZFN target site; KAN, kanamycin; hsp, heat shock promoter.

them for experiments involving the targeting of native sequences.

\section{ZFN plant expression vectors and assembly of ZFNs}

The rules and protocols for the assembly of customdesigned ZFNs have been described in several papers (e.g.
Mani et al., 2005; Wright et al., 2006) and we adapted and slightly modified the protocol described by Mani et al. (2005) for the de novo synthesis of zinc finger proteins (ZFPs). We constructed the pSAT4.35SP.NLS-Fokl vector to facilitate the assembly of a new ZFN monomer in a plant expression vector using a single cloning step. The key features of the pSAT4.35SP.NLS-Fokl vector are the presence of the Fokl endonuclease domain, a nuclear localization signal (NLS) and the Xhol and Spel recognition sites in the multi cloning site (MCS) located between the NLS and Fokl (Figure 2, ZFP cloning). The zinc finger DNA-binding domain can be cloned into the Spel and Xhol sites following its assembly from overlapping oligonucleotides by PCR, or by PCR amplification and transfer of a ZFP domain from existing ZFNs. This cloning strategy results in the construction of the pSAT4. 35SP.ZFN plant expression vector, in which the expression of a newly assembled ZFN is controlled by the CaMV $35 S$ tandem promoter (see the generic plasmid pSAT4.35SP.NLS-Fokl and its descendent pSAT4.35SP.ZFN vector in Figure 2). The $35 S$ promoter can also be easily replaced using virtually any other promoter (e.g. the heatshock promoter, hspP) in a single cloning step using Agel and $\mathrm{Ncol}$ (Figure 2, plant ZFN expression) and the entire plant expression cassette can be mounted onto pRCS-based A. tumefaciens binary vectors using rare cutters (Figure 2, binary plant transformation) for transformation experiments in plant cells.

We used pSAT4.35SP.NLS-Fokl for the construction of pSAT4.35SP.QQR and pSAT4.35SP.ZFN3, two plant expression cassettes carrying the QQR (Bibikova et al., 2001) and ZFN3 (Figure 3a) zinc finger nucleases. QQR is a well-defined ZFN which has been used in various studies (e.g. Bibikova et al., 2001; Lloyd et al., 2005) and ZFN3 is a custom-made
Figure 2. Structural features of the zinc finger nuclease (ZFN) assembly and expression vector systems.

A zinc finger protein (ZFP) coding sequence can be assembled by Klenow/PCR using a combination of overlapping backbone and sequencedependent oligonucleotides fused to the Fok endonuclease domain in pSAT4.35SP.NLS-Fokl, producing the plant expression vector pSAT4.35SP.ZFN. The entire ZFN coding sequence can be transferred onto a $\mathrm{pET} 28 . \mathrm{XH}$ based vector producing a pET28.XH-ZFN vector, suitable for ZFN expression in bacterial cells and for in vivo digestion assays. The pSAT4.35SP.ZFN can be modified by replacing the $35 \mathrm{~S}$ constitutive promoter with a heat-shockinducible promoter, producing a plasmid that is useful for the whole-plant DNA repair assay. A plant-selectable marker, a ZFN and a mutated GUS reporter expression cassette can be mounted onto a pRCS2-based binary plant transformation vector using a combination of rarecutting restriction enzymes and can then be used for various in planta assays.

35SP, 35S promoter; 35ST, 35S terminator; hsp, heat shock promoter.
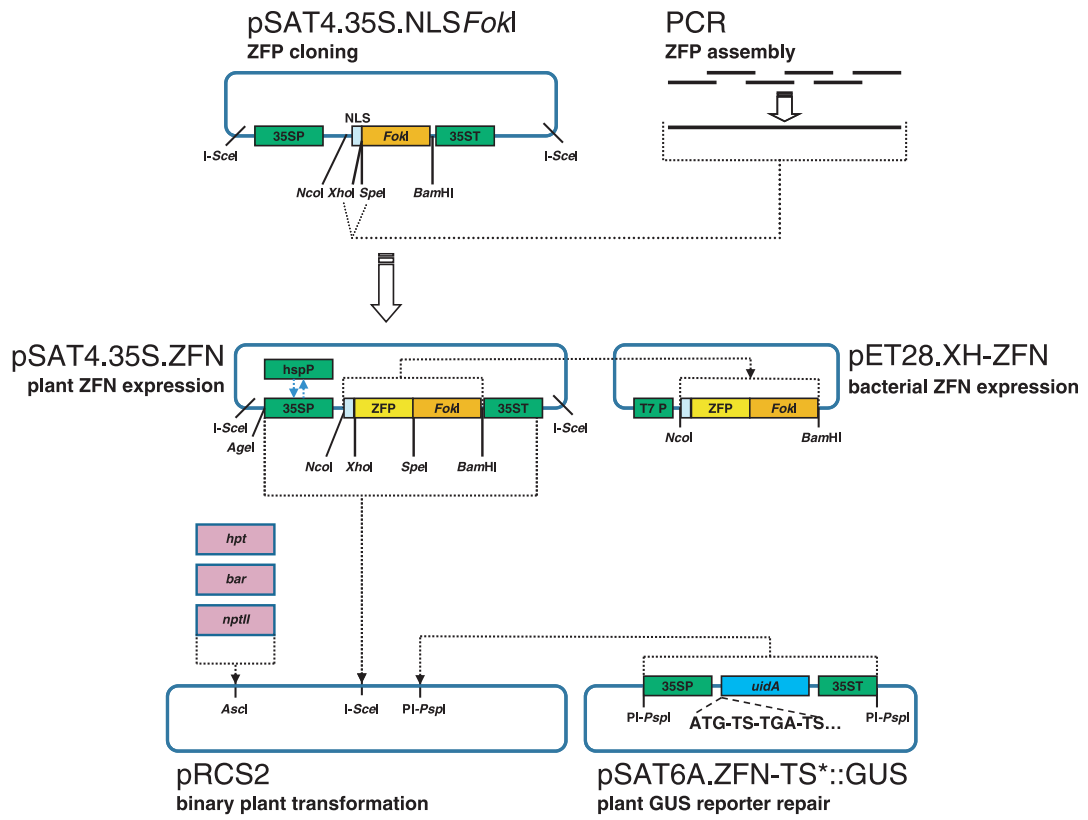
(a)
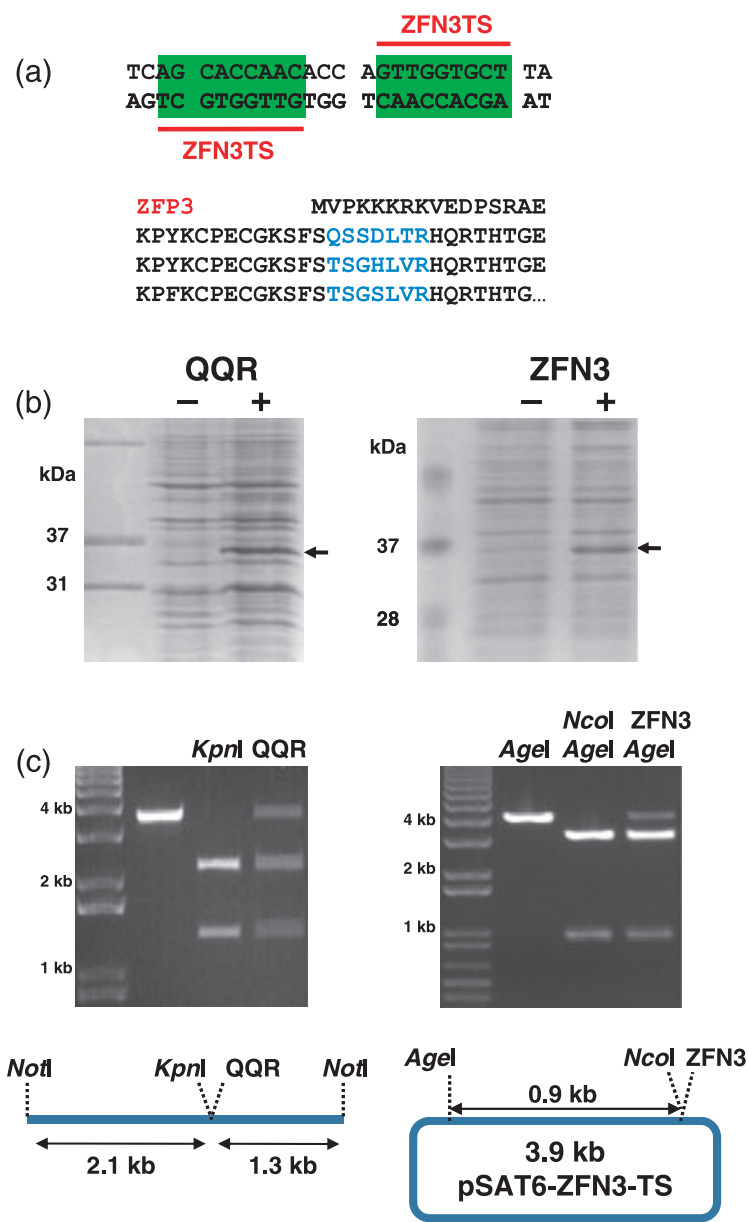

Figure 3. In-vitro digestion analysis of zinc finger nucleases (ZFNs). (a) The structure of the ZFN3 24-bp palindrome-like recognition sequence and its corresponding zinc finger protein (ZFP). ZFN3 DNA-binding sites are highlighted in green and the unique amino-acid sequences for each zinc finger are given in blue.

(b) Separation of total crude extract from isopropyl $\beta$-D-1-thiogalactopyranoside (IPTG)-induced (+) and non-induced (-) QQR and ZFN3 protein-expressing Escherichia coli cells.

(c) Restriction analysis of a 3.4-bp Notl DNA fragment carrying the QQR palindrome-like recognition sequence (left) and the pSAT6.ZFN3-TS plasmid carrying the ZFN3 palindrome-like recognition sequence (right) by their corresponding ZFNs. Also shown are the schematic maps for each target DNA plasmid.

enzyme which we designed to bind the 9-bp long sequence 5'-GGTGGTGCT (Figure 3a). We used these enzymes during the development of our in vitro and in planta digestion assays.

\section{In vitro ZFN digestion assay}

Previous studies have emphasized the importance of analyzing the in vitro cleavage activity of custom-designed ZFNs prior to their use for in vivo applications (Durai et al., 2005; Mani et al., 2005; Porteus, 2008; Wright et al., 2006). Mani et al. (2005), for example, described a protocol for the rapid characterization of ZFNs for their sequence-specific cleavage activity and constructed a set of vectors to allow the conversion of assembled ZFPs to ZFNs and for the construction of target sequences in pUC18. While these protocols and plasmids can be very useful for in vitro analysis of any given ZFN, we decided to produce our own bacterial expression vector which will be compatible with our plant vectors. We also propose a simple strategy for cloning ZFN recognition sequences into pSAT-based vectors to produce target plasmids [e.g. pSAT6.ZFN-TS, (TS, target site) Figure 1], useful for simple in vitro digestion analysis. We constructed the bacterial expression vector pET28.XH-ZFN to carry unique $\mathrm{Ncol}$ and $\mathrm{BamHI}$ sites between the T7 promoter and a 6x His tag, to allow transfer of the entire ZFN from pSAT4.35SP.ZFN plasmids in a single cloning step (Figure 2). To demonstrate our in vitro assay, we first transferred the QQR and ZFN3 from pSAT4.35SP.QQR and pSAT4.35SP.ZFN3, producing pET28.XH-QQR and pET28.XH-ZFN3, respectively. We next transferred these vectors into BL21 Escherichia coli cells for overexpression of their encoded enzymes, and we used crude bacterial lysate (Figure $3 b$ ) to assay their ability to digest their corresponding recognition sites on target plasmids. The digestion activity of QQR, expressed from our pET28.XH-QQR plasmid, was demonstrated by its ability to digest its target site which was engineered on the HS::QOR-QEQ/2300 plasmid (Lloyd et al., 2005). We released a 3.4-kb QQR target site containing Notl fragment from pHS::QQR-QEQ/2300, and its digestion with QQR released the expected 2.1- and 1.3-kb DNA fragments (Figure 3c). The 3.4-kb Notl fragment also carried a unique Kpnl recognition site next to the QQR recognition site, and its digestion with Kpnl produced a restriction pattern similar to that of QQR (Figure 3c), thus indicating that QQR indeed cleaved its corresponding site within the target fragment. It should be noted that while the digestion of the 3.4-kb Notl fragment by QQR was only partial, this does not provide conclusive evidence as to the specific activity of this enzyme since we did not purify it to the level of a commercial enzyme such as Kpnl. We further demonstrated the application of our in vitro digestion assay by analyzing the digestion activity of ZFN3. To this end, we first constructed a target plasmid, designated pSAT6.ZFN3TS, by annealing two complementary primers coding for the ZFN3 semi-palindromic target site and cloning them between the adjacent $\mathrm{Ncol}$ and Pstl sites in pSAT6-MCS. The resultant pSAT6-MCS-based vector structure (i.e. pSAT6.ZFN-TS, Figure 1) allows for single-step digestion analysis of ZFN activity by comparing the Agel/ZFN and Agel/Ncol double-digestion patterns. Indeed, the digestion pattern of pSAT6.ZFN3-TS by Agel and ZFN3 was similar to that of Agel and $\mathrm{Ncol}$ (Figure 3c), indicating that the ZFN3 can cleave its corresponding site within its target plasmid. A similar cloning strategy of annealed primers can be used to 
assemble other ZFN target sites into pSAT6-MCS, allowing for simple in vitro analysis of custom-designed ZFNs.

In planta T-DNA repair assay

Confirming the in vitro digestion activity of novel ZFNs is only the first step, albeit a crucial one, of their functional analysis in vivo. We designed a T-DNA repair assay in which the ZFN digestion activity can be analyzed by GUS staining in plant cells. The assay's first component is the plant ZFN expression cassette pSAT4.35SP.ZFN. The assay's second component is a pSAT6.ZFN-TS*::GUS plant expression cassette carrying a mutated uid $A$ gene which is engineered to carry the TGA (stop) codon within the 6-bp spacer of the ZFN target site (Figure 2, plant GUS reporter repair), leading to premature termination of uidA translation in plant cells. Digestion of the ZFN recognition site and its successive repair can lead to deletion and/or mutation of the stop codon and consequent activation of the uidA reporter gene. Assembly of the T-DNA repair assay requires the construction of a binary plasmid carrying the constitutive expression cassettes from pSAT4.35SP.ZFN and pSAT6.ZFN-TS*::GUS (i.e. the generic plasmid pRCS2.[ZFN][ZFN-TS*::GUS], Figure 1). The pSAT4.35SP.ZFN vector is based on the pSAT4 backbone plasmid that allows the entire ZFN expression cassette to be transferred onto pRCS-based binary vectors (e.g. pRCS2, Figure 2 and Tzfira et al., 2005) using I-Scel. The pSAT6.ZFN-TS*::GUS vector that carries the mutated uidA gene (ZFN-TS*::GUS) under the control of the tandem 35S promoter is based on the pSAT6 backbone plasmid (Tzfira et al., 2005), allowing the ZFN-TS*::GUS expression cassette to be transferred onto pRCS-based binary vectors using $\mathrm{PI}-p s p l$. Mounting both expression plasmids onto a single binary vector ensures efficient delivery of the tested ZFN and its target sequence into the target plant cells and allows the functional activity of the ZFN to be monitored by GUS staining.

We demonstrated the functionality of our in planta T-DNA repair assay by analyzing the activity of ZFN3 and QQR ZFNs in tobacco cells. We constructed pSAT6.ZFN3-TS*::GUS and pSAT6.QQR-TS*::GUS and mounted them and their corresponding ZFN expression cassettes (i.e. pSAT4.35SP.ZFN3 and pSAT4.35SP.QQR, respectively) onto pRCS2 binary plasmids, producing pRCS2.[ZFN3][ZFN3-TS*::GUS] and pRCS2.[QQR][QQR-TS*::GUS], respectively. The general structure of pRCS2.[ZFN3][ZFN3-TS*::GUS] and the mutated uid $A$ region which was engineered to contain the ZFN3 target site and the TGA codon is shown in Figure 4(a). Also shown in Figure 4(a) is a putative outcome of ZFN3 digestion and misrepair of the DSBs by the plant's NHEJ machinery, which can potentially lead to elimination of the stop codon and reconstruction of the uidA reporter gene. Indeed, agroinfiltration of pRCS2.[ZFN3][ZFN3-TS*::GUS] into Nicotiana benthamiana leaves resulted in positive GUS staining (a)

\section{pRCS.[ZFN3][ZFN3-TS*::GUS]}
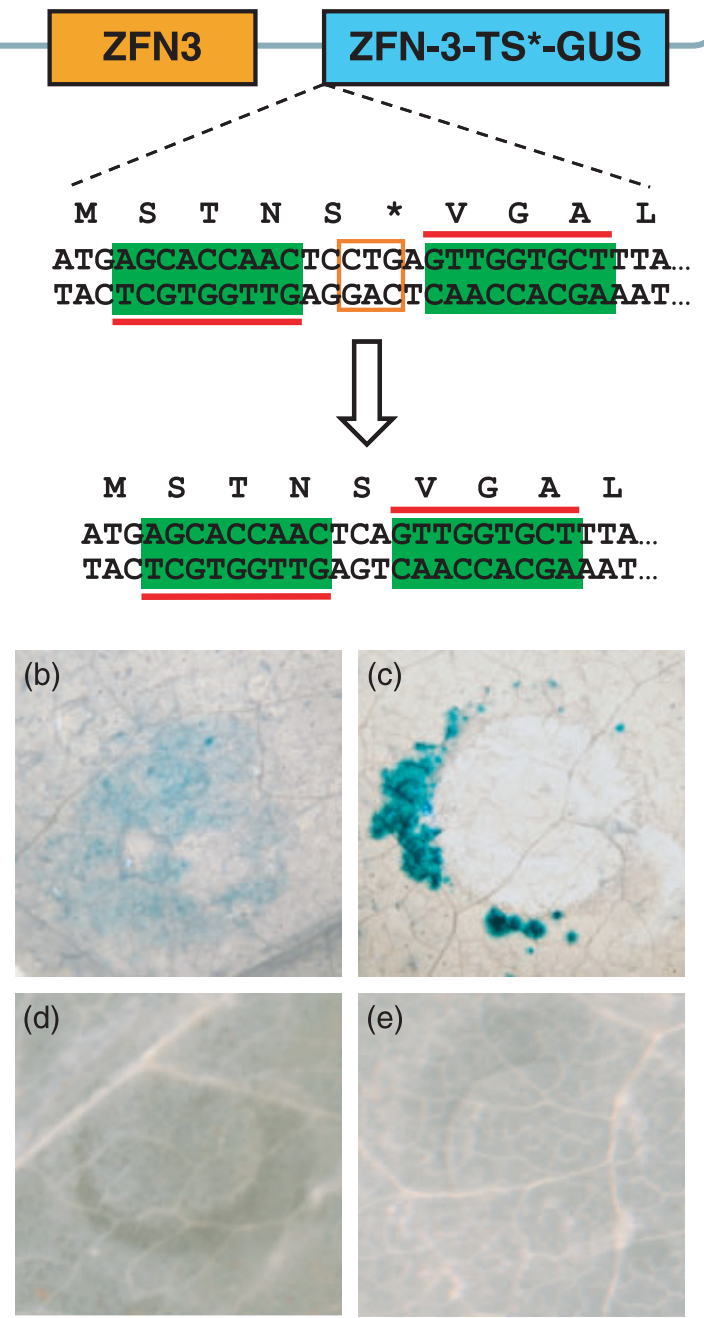

Figure 4. A T-DNA repair assay in tobacco leaves.

(a) The structure of a T-DNA repair assay plasmid is exemplified by a binary plasmid constructed to test ZFN3 activity in plant cells. Reconstruction of a mutated GUS-encoding gene is exemplified by putative deletion of a CTG sequence (in the orange box) which may occur following the digestion and misrepair of the ZFN3 recognition site in planta.

(b-e) Transformed tobacco leaves infected with QQR (b, d) or ZFN3 (c, e) mutated GUS expression cassettes, with $(b, c)$ or without $(d, e)$ their corresponding digesting zinc finger nucleases on the T-DNA molecule.

(Figure 4b), indicating the functional activity of the ZFN3 protein in plant cells. Agroinfiltration of pRCS2.[QQR][QQRTS*::GUS] also resulted in positive GUS staining, although with higher intensity (Figure 4c), which may be attributed to the higher DNA-binding affinity of QQR relative to that of ZFN3. Expression of GUS was not observed following agroinfiltration of binary plasmids carrying the ZFN3-TS*::GUS (Figure 4d), or QQR-TS*::GUS (Figure 4e) 
expression cassettes without their corresponding ZFN expression cassettes, indicating that ZFN expression is required for digestion and activation of the uid $A$ reporter gene from the $A$. tumefaciens T-DNA in planta.

\section{In planta transgene repair assay}

The ability of ZFNs to digest T-DNA molecules in plant cells provides another level of confidence for their potential use in living cells. Nevertheless, we recommend testing the ability of ZFNs to digest genomically embedded DNA sequences prior to their deployment for GT experiments, as suggested by other studies aimed at developing ZFNs for non-plant species (e.g. Cathomen et al., 2008; Porteus, 2008). To this end, we designed the transgene repair assay in which the ZFN activity is tested on a mutated uidA expression cassette which was first integrated into the genome of transgenic tobacco calli. The assay's first component is a binary transformation vector that carries the ZFN-TS*::GUS reporter expression cassette and a selectable marker (e.g. npt/l) (Figure 1, generic plasmid pRCS2.[KAN][ZFN-TS*::GUS]). This vector can be used to produce transgenic calli which lack functional uidA translation. The second component is a binary vector, carrying the ZFN expression cassette, driven by the tandem 35S promoter (Figure 1, generic plasmid, pRCS2.[ZFN]). This vector is used for infection of the transgenic calli and for digestion of the ZFN-TS*::GUS expression cassette in these tissues.

We demonstrated the functionality of our transgene repair assay by testing the activity of ZFN3 and QQR ZFNs in tobacco cells. We constructed pRCS2.[KAN][ZFN3TS*::GUS] and pRCS2.[KAN][QQR-TS*::GUS] and used them for the production of kanamycin-resistant transgenic tobacco calli. As expected, these transgenic calli did not express functional uidA, as indicated by their negative GUS staining (Figure 5a,b). We next constructed pRCS2.[ZFN3] and pRCS2.[QQR] and used them in wounding-infection experiments with the ZFN3-TS*::GUS and QQR-TS*::GUS transgenic calli, respectively, which resulted in positive GUS staining (Figure $5 c, d$ ), indicating the functionality of these ZFNs in digesting integrated DNA molecules.

\section{A whole-plant DNA repair assay}

The in planta transgene repair assay provides clear experimental evidence of the digestion activity of the analyzed ZFNs on genomically integrated DNA sequences. We next developed a whole-plant DNA repair assay as a tool for analyzing, at the molecular level, the outcome of their digestion and the repair of the digested DNA in transgenic plants. The assay calls for the assembly of a multi-gene plant transformation vector, which carries a plant-selectable marker, the mutated uidA reporter gene cloned under the control of a constitutive promoter and the ZFN protein
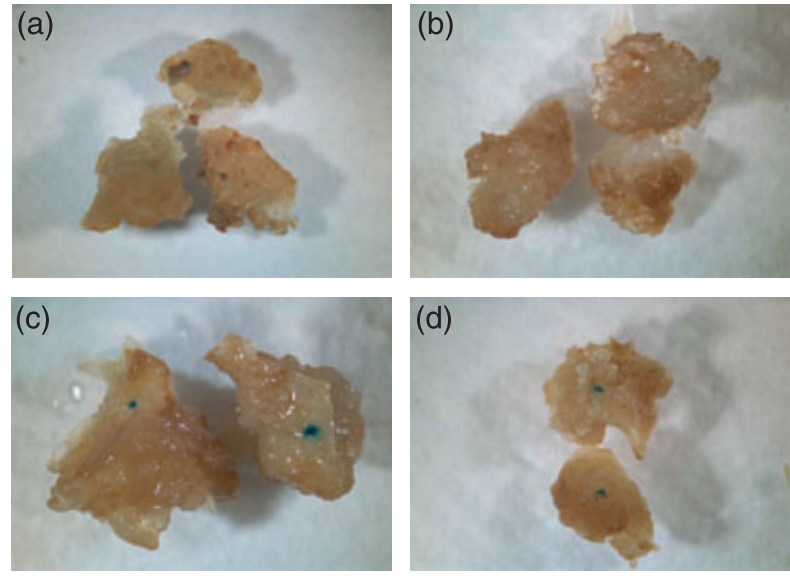

Figure 5. Transgene repair assay in tobacco calli.

(a, b) X-Gluc-stained kanamycin-resistant calli carrying mutated GUS-encoding sequences engineered with a QQR (a) or ZFN3 (b) target site.

(c, d) Detection of mutated GUS-encoding sequence activation by X-Gluc staining of transgenic calli engineered with a QQR (c) or ZFN3 (d) target site and inoculated with their corresponding zinc finger nucleases.

cloned under the control of an inducible promoter (Figure 1, pRCS2.[KAN][hspP.ZFN][ZFN-TS*::GUS] generic plasmid). We used the Arabidopsis heat-shock-inducible promoter HSP18.2 (Takahashi and Komeda, 1989), but other types of inducible promoters may be considered. The vector can be used to produce transgenic plants and to monitor ZFN activity in various tissues at different developmental stages. We used this assay to monitor the activity of QQR ZFN in different tissues of Arabidopsis transgenic plants and to molecularly characterize the outcome of various uidA digestion and repair events in these plants. To this end, we constructed pRCS2.[KAN][hspP.QQR][QQR-TS*::GUS], produced several kanamycin-resistant Arabidopsis plants, and used heat shock to induce the expression of the QQR enzyme in mature plants and their offspring. Induction of ZFNs in fully developed mature transgenic leaves resulted in activation of the uidA reporter gene, as indicated by the appearance of GUS-expressing spots on the induced leaves (Figure 6a). Induction of ZFNs in transgenic seeds resulted in the appearance of GUS-expressing sectors (Figure 6b) and the production of chimeric tissues (e.g. Figure $6 \mathrm{c}$ ). We allowed a few of the heat-shocked transgenic seedlings to develop, stained them and found that they exhibited extensive GUS staining (e.g. Figure 6d). No GUS expression was observed in non-induced transgenic plants.

We further analyzed several samples from ZFN-induced transgenic seedlings by PCR amplification of the mutated QQR-TS*::GUS region. While repair of DSBs induced by restriction enzymes has been shown to create various sitespecific mutations, including large deletions and/or insertions at the break site (Kirik et al., 2000; Lloyd et al., 2005; Salomon and Puchta, 1998), we focused our analysis on 

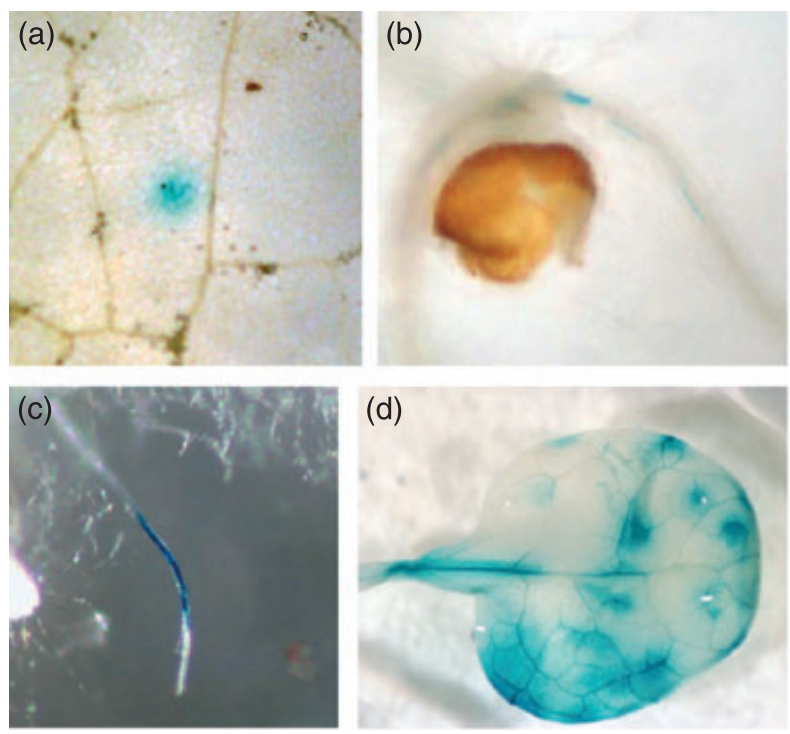

(d)

Figure 6. Whole-plant DNA repair assay in Arabidopsis.

Detection of zinc finger nuclease activity in mature leaves (a), germinating seedlings (b), elongating root (c) and developing leaves (d) of transgenic Arabidopsis plants engineered to carry a heat-shock-inducible QQR and its corresponding mutated GUS-encoding sequence.

DdeI

ATGTTCTTCСССТССTGAGGGGAAGAATTA
$\begin{array}{llllllllll}\text { M } & \text { F } & \text { F } & \text { P } & \text { S } & \bullet & \text { G } & \text { E } & \text { E } & \text { L }\end{array}$

ATGTTCTTCCCCTCCCGAGGGGAAGAATTA ATGTTCTTCCCCTCCAGAGGGGAAGAATTA ATGTTCTTCCССTCCTGGGGGGAAGAATTA ATGTTCTTCCССTCCTGGAGGGGAAGAATTA ATGTTCTTCCCCTCCTG-GGGAAGAATTA ATGTTCTTCCC-----GAGGGGAAGAATTA ACGAAC . . . -82. bP. . . . TGTAGA

Figure 7. Molecular analysis of zinc finger nuclease (ZFN)-induced mutations in transgenic Arabidopsis plants.

The ZFN-binding sites on the upper strands of the mutated GUS-encoding sequence are highlighted in green. The location of the $D d e l$ recognition site is indicated by a red bar. The outcome of positive $(+)$ or negative (-) GUS expression in the different types of ZFN-mediated deletions or substitutions is indicated on the left.

identifying small, single-nucleotide deletions and/or substitutions in the mutated open reading frame (ORF) which could lead to reconstruction of an active uidA gene. To this end, we first digested the total DNA extract of the induced plants with Ddel in order to reduce the contamination with non-mutated CTGAG-sequence DNA. We next used PCR to amplify the undigested DNA, cloned the PCR products and analyzed them by DNA sequencing. Figure 7 shows the sequencing results of some of these clones, which revealed the presence of nucleotide replacement and small deletions and/or additions, some of which can explain the reconstruction of a functional uidA gene. This analysis can thus be used to predict at least part of the possible outcome of ZFN-mediated GT in plant cells.

\section{Discussion}

Zinc finger nucleases have been proven instrumental for genome editing in various species. Expression of model and tailor-made ZFNs in model organisms such as Drosophila, Caenorhabditis, Xenopus, Danio and human cell lines has been shown to lead to various outcomes of genome editing. These include site-specific mutagenesis, gene replacement by $\mathrm{HR}$, and targeted gene addition at specific locations (e.g. Beumer et al., 2006; Bibikova et al., 2001; Carroll et al., 2008; Doyon et al., 2008; Meng et al., 2008; Moehle et al., 2007; Morton et al., 2006; Santiago et al., 2008; Urnov et al., 2005). Zinc finger nucleases have also been shown to enhance HR and to lead to site-specific mutagenesis in Arabidopsis and tobacco model plants (Lloyd et al., 2005; Wright et al., 2005); more recently, Dow AgroSciences and Sangamo BioSciences announced the modification of maize and canola genomes by ZFNs (http://files.shareholder.com/downloads/ SGMO/0x0x113031/d58602ac-aed0-4118-8955-e98e4004aa9 d/SGMO_News_2007_6_19_General_Releases.pdf). Thus, ZFNs represent a novel and powerful technology which may eventually lead to the development of much needed GT methods for plant species (Tzfira and White, 2005).

The application of ZFN technology for the GT of native sequences requires several technological barriers to be overcome. Proper selection of the binding site for a pair of ZFN monomers on the target sequence is the first step in the process of using this technology for genome editing in living cells. The rules for selecting target sites have been described in several excellent papers (Mandell and Barbas, 2006; Porteus, 2008; Sander et al., 2007) and the developer of novel ZFNs can also use a collection of data sets describing the DNA-binding domains for 49 out of 64 possible triplets (Mandell and Barbas, 2006), as well as publicly available web-based programs (http://www.zincfingers.org/ and http://www.zincfingertools.org) to assist with the design of novel ZFNs. Once designed, the developer is faced not only with the challenge of assembling the ZFNs but also with validating their activity in vitro and in vivo, prior to their deployment for genome editing experiments in their target organism. Various cloning methods can potentially be used for the assembly of customized ZFNs. Restriction-digestbased modular assembly, for example (Wright et al., 2006), is based on successive cloning of individual fingers from a collection of finger modules and the production of a multifinger construct which can then be used for the assembly of custom-made ZFNs. In another strategy (Porteus, 2008), several fingers are first independently amplified using a combination of general and finger-specific primers and then 
assembled together via a second overlapping PCR step. We adopted the strategy of de novo assembly (Mani et al., 2005) of custom-designed ZFPs from a collection of overlapping backbone and sequence-dependent oligonucleotides (Figure 2, ZFP assembly). This strategy, which is based on annealing and ligating the assembled oligonucleotides followed by PCR amplification of the resultant fragment, allows the construction of novel ZFP domains in a single reaction. To facilitate the use of this strategy for the assembly of novel ZFNs for plant research, we constructed pSAT4.35S.NLS-Fokl (Figure 2, ZFP cloning), which allows for single-step fusion of a ZFP with a NLS and the Fokl endonuclease domain, producing pSAT4.35SP.ZFN (Figure 2, plant ZFN expression). No less important was the construction of the pET28.XH bacterial expression vector (Figure 2, bacterial ZFP expression) which allows the simple transfer of ZFN coding sequences from pSAT4.35S.ZFN. The pSAT4.35SP.ZFN and pET28.XH-ZFN expression vectors are the key plasmids for the in vivo analysis of newly assembled ZFNs.

Dedicated bacterial expression of ZFN vectors for in vitro analysis of custom-designed ZFNs has been described in several reports. The pET15b:N vector, for example (Mani et al., 2005), was constructed for in vitro transcriptiontranslation of ZFNs and their validation in conjunction with pUC18-based target sequences, and the pMAL-c2X-based bacterial expression vector was designed for maltose-binding protein (MBP) tagging of ZFNs (Cathomen et al., 2008). The latter enables protein purification on amylase resin and the use of purified ZFNs in in vitro activity analysis on target DNA sequences. We found that cell lysates (Figure $3 b$ ) from ZFN-expressing $E$. coli cells using our modified pET28.XH vector were useful for in vitro digestion analysis of target DNA molecules (Figure 3c). Thus, while the use of an in vitro expression method and/or epitope-based extraction may produce higher protein concentrations and/or a more highly purified product, simple crude protein extraction may also be sufficient for analysis of newly assembled ZFNs. Furthermore, while in vitro digestion analysis is typically performed by sequential digestion of target DNA molecules using ZFNs and commercial enzymes (e.g. Cathomen et al., 2008; Mani et al., 2005), we found that double digestion can be performed in a single reaction, which greatly simplifies the in vitro validation process. The qualitative nature of our in vitro digestion assay does not allow us to quantify the specific activity of ZFNs, but it is sufficient for determining their functionality.

In vitro digestion analysis is just the first step in validating ZFN activity for in vivo applications. Two basic approaches have been developed to assay novel ZFNs in living cells. The first approach is based on analyzing the binding activity of the DNA-binding domain of the ZFNs, and it has been adapted, for example, by Wright et al. (2006), who developed a two-hybrid reporter system for analysis of the binding activity of ZFPs in bacterial cells. In their system, expression of the ZFP-Gal11P and alpha-Gal4 hybrid proteins led to activation of the lacZ reporter gene in bacterial cells upon binding of the ZFP with its target site, which was engineered upstream of the lac $Z$ promoter. In another report, Meng et al. (2008) developed a one-hybrid activation system in which fusion of ZFP to RNA polymerase resulted in activation of the HIS and URA genes in bacterial cells. Both systems offer a robust, rapid and easily performed assay for analyzing ZFP-binding activity, but they do not allow for direct evaluation of the ZFN digestion activity in target cells. The second approach overcomes this limitation since it is based on analyzing the digestibility of ZFN, and not only binding, in living cells. One such system was recently described by Doyon et al. (2008), who developed a yeastbased chromosomal reporter system in which the activity of novel ZFNs could be measured following the repair of a ZFNmediated DSB in the yeast genome. Building on the notion that the digestion of tester sequences may provide more reliable information on the potential activity of newly assembled ZFNs in digesting native sequences in living cells, we adapted the second approach during the construction of our reporter ZFN-validation assays for plant cells.

Zinc finger nuclease-mediated DSBs of episomal or chromosomally embedded or encoded target sequences can potentially be repaired by HR or lead to enhanced HR at the break site. Nevertheless, genomic DSBs in many organisms, and particularly in plant species, are typically repaired by the cell's NHEJ machinery. Misrepair of broken edges can lead to deletions, insertions and/or substitutions and even capture of foreign DNA molecules at the repaired site. This wide array of possible outcomes could potentially hinder the efficiency of our reporter gene repair assay which can tolerate only short, in-frame deletions and/or alterations of a few nucleotides at the mutated uidA internal stop codon. Nevertheless, we have clearly shown that our in planta repair assays are sensitive enough to detect proper activation of the mutated uidA gene. This was further supported by molecular analysis of several repair events produced by a whole-plant DNA repair assay which revealed the reconstruction of a functional uidA gene (Figure 7).

Several key features of our plant-specific ZFN-validation assays make them useful for simple and robust analysis of custom-made ZFNs. First, our vector system is based on the structure of our previously described pSAT plant expression vector system which was specifically designed to facilitate the assembly of multi-gene expression cassettes (Tzfira et al., 2005). This feature is exemplified during the construction of the T-DNA repair assay, in which both the ZFN and the mutated GUS expression cassettes were mounted onto a single binary plasmid using a pair of rare-cutters which was then used in a single transient transformation experiment (Figure 4). Second, our gene-activation assays are based on the uidA gene encoding the sensitive GUS enzyme. This 
allows one to monitor ZFN activity using a simple biochemical staining procedure, which has been reported as useful in a wide range of plant species, tissues and cell types (Jefferson et al., 1987). We should also note that while the expression of ZFNs is driven by the tandem $35 \mathrm{~S}$ promoter in our pSAT-based vectors, the promoter and terminator regions can easily be replaced by other regulatory elements (Chung et al., 2005; Dafny-Yelin and Tzfira, 2007) which may be more suitable for the ZFN developer's species of choice. This feature is exemplified during the conversion of pSAT4.35SP.QQR to pSAT4.hspP.QQR and the use of the latter plasmid for heat-shock induction of the QQR ZFN in transgenic Arabidopsis plants (Figure 6). Third, our transgene repair and whole-plant DNA repair assays, which call for the assembly of $A$. tumefaciens stable transformation vectors and the recovery of transgenic tissues and/or whole plants (Figures 5 and 6), can support the use of different selection genes (Figure 2 and Chung et al., 2005; DafnyYelin and Tzfira, 2007), providing the user with greater flexibility in the choice of selectable marker. Fourth, our set of vectors provides the user with a variety of options for analyzing the digestive activity of novel ZFNs. Thus, while we recommend a systematic analysis of novel ZFNs before their deployment for targeting experiments, users of our system can choose to analyze their ZFNs at different levels of confidence, depending on their choice of assay.

In summary, we describe the construction and experimental application of a new set of assays and supporting plasmids for the validation of ZFN activity in plant cells. These complement a long line of assays and vectors which have been specifically designed to facilitate the assembly, analysis and use of ZFNs in non-plant species. Given the simplicity of our assay system, its cloning flexibility and its compatibility with a much larger array of vectors carrying a large selection of promoters, terminators, selectable markers and reporter genes (Chung et al., 2005; Dafny-Yelin and Tzfira, 2007; Tzfira et al., 2005), our hope is that this system will facilitate experimentation with ZFN technology in plant species.

\section{Experimental procedures}

\section{DNA constructs}

Standard DNA amplification and cloning methods were used during the construction of all our vectors, unless noted otherwise. To produce a plant ZFP cloning vector that carries a NLS and the Fokl endonuclease domain, we first cloned the NLS coding sequence by annealing the 5'-CATGCCATGGTGCCAAAAAAGAAGAGAAAGGTAGAAGACCCCTCTCGAGCGG-3' and 5'-CCGCTCGAGAGGGGTCTTCTACCTTTCTCTTCTTTTTTGGCACCATGGCATG-3' primers and cloning the DNA product as a Ncol-Xhol fragment into pSAT6-MCS (Tzfira et al., 2005), producing pSAT6.35SP.NLS-MCS. We next PCRamplified the Fokl endonuclease domain from pHS::QQR-QEQ/2300 (Lloyd et al., 2005) using the 5'-ACGCGTCGACGGACTAGTCAAAAGTGAACTGG-3' and 5'-GAAGATCTTTAGGATCCAAAGTTTATCT-
CGCCGTTATTAAA- $3^{\prime}$ primers and cloned it as a Sall-Bgll fragment into the Sall and BamHI sites of pSAT6.35SP.NLS-MCS, producing pSAT6.35SP.NLS-Fokl. Finally, we transferred the NLS-Fokl expression cassette as a Agel-Notl fragment from pSAT6.35SP. NLS-Fokl into pSAT4-MCS (Tzfira et al., 2005), producing pSAT4.35SP.NLS-Fokl. To produce pET28.XH we first annealed the 5'-AACTTTGGATCCCTCGAGTAGGACTGCAGGCTTGCGGCCGCACTCGA-3' and 5'-TCGAGTGCGGCCGCAAGCCTGCAGTCCTACTCGAGGGATCCAAAGTT-3' primers and cloned the DNA product as a BamHI-Notl fragment into the $\mathrm{pET} 28 \mathrm{c}(+)$ bacterial expression vector (Invitrogen, http://www.invitrogen.com/) producing the intermediate plasmid pET28.XX. We next removed the spacer between the $\mathrm{BamHl}$ site and the $6 \times$ His coding sequence by cleaving $\mathrm{pET} 28 . \mathrm{XX}$ at two Xhol sites and self-ligating it. The resultant pET28.XH contained unique $\mathrm{Ncol}$ and BamHI sites, allowing the assembly of a ZFN::6x His fusion in a single cloning step.

To produce a constitutive plant expression cassette for the ZFN $\mathrm{QQR}$, we PCR-amplified the QQR coding sequence from pHS::QQR$\mathrm{QEQ} / 2300$ and cloned it as a Ncol-BamHI fragment into pSAT4.35SP.NLS-Fokl, producing pSAT4.35SP.QQR. To produce a heat-shock-inducible plant expression cassette for QQR, we PCRamplified the HSP18.2 promoter sequence from pHS::QQR-QEQ/ 2300 and cloned it into the Agel-Ncol sites of pSAT4.35SP.QQR, replacing the tandem CaMV 35 S promoter with an HSP18.2 promoter, and producing pSAT4.hspP.QQR. To produce a constitutive plant expression cassette for ZFN3, the ZFP was first assembled from a set of overlapping backbone oligonucleotides (BBO primers: 5'-GAAAAACCTTACAAGTGTCCTGAATGTGGAAAGTCTTTTTCT-3', 5'-CAGCGAACACACACAGGTGAGAAGCCATATAAATGCCCAGAATGTGGTAAATCATTCAG-3' and 5'-CAACGGACCCAC ACCGGGGAGAAGCCATTTAAATGCCCTGAGTGCGGGAAGAGTTTTT-3') and sequence-dependent oligonucleotides (SDO primers: $5^{\prime}$ ACCTGTGTGTGTTCGCTGGTGACGAGTAAGATCAGAAGACTGAGAAAAAGACTTTCCACA-3', 5'-CCCGGTGTGGGTCCGTTGGTGACG AACAAGATGTCCAGAAGTACTGAATGATTTACCACA-3' and 5'-TC CAGTATGAGTACGTTGATGACGAACAAGAGATCCAGAAGTTGAAAAACTCTTCCCGCAC-3') as previously described (Mani et al., 2005) using high-fidelity Pfu DNA polymerase (Stratagene, http:// www.stratagene.com/). The PCR mixture, consisting of $5 \mu \mathrm{m}$ each of BBO and SDO primers and $200 \mu \mathrm{m}$ each of $5^{\prime}$-CCGCTCGAGCTGAAAAACCTTACAAGTGTCC- $3^{\prime}$ and $5^{\prime}$-GGACTAGTCCTCCAGTATGAGTACGTTGATG-3' primers, was carried out for 35 cycles. The PCR product was cloned into the $\mathrm{Xhol}$ and Spel sites of pSAT4.35S.NLS-Fokl, producing pSAT4.35SP.ZFN3.

For expression of ZFN in E. coli cells, the QQR and ZFN3 coding sequences were transferred from pSAT4.35SP.QQR and pSAT4.35SP.ZFN3 as Ncol-BamHI fragments and cloned into pET28.XH, producing pET28.XH-QQR and pET28.XH-ZFN3, respectively. The ZFN3 target plasmid pSAT6.ZFN3-TS was constructed by annealing the 5 '-CGATAGCCATGGAGCACCAACACCAGTTGGTGCTCTGCAGTCGACG-3' and 5'-CGTCGACTGCAGAGCACCAACTGGTGTTGGTGCTCCATGGCTATCG-3' primers and cloning the DNA product as a Ncol-Pstl fragment into pSAT6-MCS. To produce mutated GUS-expression vectors carrying the QQR zinc finger recognition sites, the uidA coding sequence was PCR-amplified from pRTL2-GUS using the forward 5'-GGGGTACCATGTTCTTCCCCTCCTGAGGGGAAGAATTACGTCCTGTAGAAACCCC- $3^{\prime}$ and reverse 5'-CGGGGTACCATGTTACGTCCTGTAGAAACCCC-3' primers and cloning the PCR product into the Kpnl-BamHI sites of pSAT6A-MCS (Chung et al., 2005), producing pSAT6A.QQRTS*::GUS. A similar strategy was used to construct pSAT6A.ZFN3TS*::GUS, using the forward $5^{\prime}$-GGGGTACCATGAGCACCAACTCCTGAGTTGGTGCTTTACGTCCTGTAGAAACCCC- $3^{\prime}$ and the above-mentioned reverse primer. 
The binary vectors pRCS2.[ZFN3-TS*::GUS] and pRCS2.[QQR$\mathrm{TS}^{*}:: \mathrm{GUS}$ ] were constructed by transferring the ZFN3-TS*::GUS and QQR-TS*::GUS expression cassettes from pSAT6A.ZFN3-

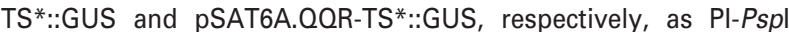
fragments into pRCS2 (Tzfira et al., 2005). The binary vectors pRCS2.[ZFN3][ZFN3-TS*::GUS] and pRCS2.[QQR][QQR-TS*::GUS] were constructed by transferring the ZFN3 and QQR constitutive expression cassettes from pSAT4.35SP.ZFN3 and pSAT4.35SP.QQR as I-Scel fragments into pRCS2.[ZFN3-TS*::GUS] and pRCS2.[QQRTS*::GUS], respectively. The binary vectors pRCS2.[KAN][ZFN3TS*::GUS] and pRCS2.[KAN][QQR-TS*::GUS] were constructed by transferring the ZFN3-TS*::GUS and QQR-TS*::GUS expression cassettes from pSAT6A.ZFN3-TS*::GUS and pSAT6A.QQRTS*::GUS, respectively, as PI-Pspl fragments into pRCS2-nptll (Chung et al., 2005). The binary vectors pRCS2.[ZFN3] and pRCS2.[QQR] were produced by removing the ZFN3-TS*::GUS and QQR-TS*::GUS expression cassettes from pRCS2.[ZFN3][ZFN3TS*::GUS] and pRCS2.[QQR][QQR-TS*::GUS], respectively. The pRCS2.[KAN][hspP.QQR][QQR-TS*::GUS] binary vector was constructed by cloning the heat-shock-induced $\mathrm{QQR}$ expression cassette from pSAT4.hspP.QQR into pRCS2.[QQR-TS*::GUS].

\section{Zinc finger nuclease protein expression and in vitro digestion assay}

For E. coli expression, ZFN expression plasmids (pET28.XH-ZFN3 and pET28.XH-QQR) were transformed into BL21 GOLD (DE3) PlyS cells (Stratagene). The cells were cultured in $100 \mathrm{ml}$ of LB medium supplemented with $50 \mu \mathrm{g} \mathrm{ml}^{-1}$ kanamycin and $100 \mu \mathrm{M} \mathrm{ZnCl}$ and grown at $22^{\circ} \mathrm{C}$. At an $\mathrm{OD}_{600}$ of 0.6 , ZFN expression was induced by $0.7 \mathrm{~mm}$ isopropyl $\beta$-D-1-thiogalactopyranoside (IPTG) for $3 \mathrm{~h}$ at $22^{\circ} \mathrm{C}$. Cells were harvested by centrifugation for $10 \mathrm{~min}$ at $3000 \mathrm{~g}$ resuspended in $35 \mathrm{ml}$ of $25 \mathrm{~mm}$ 2-amino-2-(hydroxymethyl)1,3propanediol (TRIS)- $\mathrm{HCl} \mathrm{pH} \mathrm{7.5,} 300 \mathrm{~mm} \mathrm{NaCl}, 5 \%$ (v/v) glycerol and $100 \mu \mathrm{M} \mathrm{ZnCl}$ and lysed twice using a French Press. The cell lysates were used directly for target-site plasmid cutting in the biochemical assay. Digestion of the target-site plasmids or DNA fragments was carried out in NE Buffer 4 (New England Biolabs, http://www.neb. $\mathrm{com} / /$ for $30 \mathrm{~min}$ at $37^{\circ} \mathrm{C}$ using $0.25-2.5 \mu \mathrm{l}$ of cell lysates. Doubledigestion with ZFN3 and Agel was performed by combining both enzymes in the same reaction mix.

\section{Plant transformation}

Binary vectors were transferred into $A$. tumefaciens strain EHA105. For the T-DNA repair assay, $A$. tumefaciens cells were grown overnight at $28^{\circ} \mathrm{C}$ in LB medium supplemented with spectinomycin and streptomycin $\left(200 \mu \mathrm{g} \mathrm{ml}^{-1}\right.$ each), collected by centrifugation for 10 min at $3000 \mathrm{~g}$ and resuspended in an induction medium $\left[10.5 \mathrm{~g} \mathrm{~L}^{-1}\right.$ $\mathrm{K}_{2} \mathrm{HPO}_{4}, 4.5 \mathrm{~g} \mathrm{~L}^{-1} \mathrm{KH}_{2} \mathrm{PO}_{4}, 1 \mathrm{~g} \mathrm{~L}^{-1}\left(\mathrm{NH}_{4}\right)_{2} \mathrm{PO}_{4}, 0.5 \mathrm{~g} \mathrm{~L}^{-1}$ sodium citrate, $1 \mathrm{~g} \mathrm{~L}^{-1}$ glucose, $1 \mathrm{~g} \mathrm{~L}^{-1}$ fructose, $4 \mathrm{~g} \mathrm{~L}^{-1}$ glycerol, $120 \mathrm{mg} \mathrm{L}^{-1}$ $\mathrm{MgSO}_{4}, 2 \mathrm{~g} \mathrm{~L}^{-1}$ 2-( $\mathrm{N}$-morpholine)-ethanesulfonic acid (MES), $\mathrm{pH}$ 5.6] supplemented with $100 \mu \mathrm{m}$ acetosyringone and antibiotics, grown to an $\mathrm{OD}_{600}$ of 0.6 , again collected by centrifugation for 10 min at $3000 \mathrm{~g}$ and resuspended in an infiltration medium (10 mM $\mathrm{MgSO}_{4}, 10 \mathrm{~mm}$ MES, pH 5.6) supplemented with $200 \mu \mathrm{M}$ acetosyringone. The Agrobacterium infiltration method (Van der Hoorn et al., 2000) and intact tobacco leaves were used for the delivery of T-DNA molecules for the T-DNA repair assay. The leaf-disk transformation method (Gallois and Marinho, 1995) with only a callusinduction medium instead of a shoot-regeneration medium was used for the production of transgenic calli for the in planta T-DNA repair assay using the pRCS2.[KAN][ZFN3-TS*::GUS] or
pRCS2.[KAN][QQR-TS*::GUS] vectors. For activation of the uidA gene in the in planta T-DNA repair assay, transgenic calli were reinfected with $A$. tumefaciens cells carrying the appropriate binary vectors (i.e. pRCS2.[ZFN3] or pRCS2.[QQR]) for $48 \mathrm{~h}$ at $25^{\circ} \mathrm{C}$ on a hormone-free MS medium. For the whole-plant DNA repair assay, Arabidopsis plants were transformed using the floral dip transformation method (Zhang et al., 2006), and transgenic plants, tissues and/or seedlings were heat-shocked at $42^{\circ} \mathrm{C}$ for $30 \mathrm{~min}$. Infected leaves, transgenic calli and heat-shocked leaves and seedlings were stained with chromatogenic substrate X-Gluc as described previously (Jefferson et al., 1987).

\section{Molecular analysis of mutagenized transgenic Arabidopsis plants}

Total plant DNA was extracted from leaves of heat-shocked transgenic Arabidopsis plants using the phenol-chloroform method. Total DNA was digested with Ddel for $1 \mathrm{~h}$ and the region surrounding the ZFN target site was PCR-amplified using primers 5'-CATGCCATGGCTATCCTTCGCAAGACCCTTCC- $3^{\prime}$ and 5'-GAC TAGTGAGCATTACGCTGCGATG- $3^{\prime}$. The resulting PCR fragment was redigested with Ddel, and its undigested fraction was reamplified and cloned as an $\mathrm{Ncol}$-Spel fragment into pSAT5-MCS (Tzfira et al., 2005). Randomly selected colonies were then selected and sequenced. All genetic materials are available on request.

\section{Acknowledgements}

AT is a pre-doctoral student in the Graduate Program in Genetics, Stony Brook University, Stony Brook, NY, USA. We thank Dr G. N Drews for the gift of $\mathrm{pHS}:$ :QQR-QEQ/2300. This work was supported by University of Michigan startup funds.

\section{References}

Beumer, K., Bhattacharyya, G., Bibikova, M., Trautman, J.K. and Carroll, D. (2006) Efficient gene targeting in Drosophila with zincfinger nucleases. Genetics, 172, 2391-2403.

Bibikova, M., Carroll, D., Segal, D.J., Trautman, J.K., Smith, J., Kim, Y.G. and Chandrasegaran, S. (2001) Stimulation of homologous recombination through targeted cleavage by chimeric nucleases. Mol. Cell. Biol. 21, 289-297.

Britt, A.B. and May, G.D. (2003) Re-engineering plant gene targeting. Trends Plant Sci. 8, 90-95.

Carroll, D. (2004) Using nucleases to stimulate homologous recombination. Methods Mol. Biol. 262, 195-207.

Carroll, D., Morton, J.J., Beumer, K.J. and Segal, D.J. (2006) Design, construction and in vitro testing of zinc finger nucleases. Nat. Protoc. 1, 1329-1341.

Carroll, D., Beumer, K.J., Morton, J.J., Bozas, A. and Trautman, J.K. (2008) Gene targeting in Drosophila and Caenorhabditis elegans with zinc-finger nucleases. Methods Mol. Biol. 435, 63-77.

Cathomen, T., Segal, D.J., Brondani, V. and Muller-Lerch, F. (2008) Generation and functional analysis of zinc finger nucleases. Methods Mol. Biol. 434, 277-290.

Chilton, M.-D.M. and Que, Q. (2003) Targeted integration of T-DNA into the tobacco genome at double-strand breaks: new insights on the mechanism of T-DNA integration. Plant Physiol. 133, 956965.

Chiurazzi, M., Ray, A., Viret, J.F., Perera, R., Wang, X.H., Lloyd, A.M. and Signer, E.R. (1996) Enhancement of somatic intrachromoso- 
mal homologous recombination in Arabidopsis by the $\mathrm{HO}$ endonuclease. Plant Cell, 8, 2057-2066.

Chung, S.M., Frankman, E.L. and Tzfira, T. (2005) A versatile vector system for multiple gene expression in plants. Trends Plant Sci. 10, 357-361.

Dafny-Yelin, M. and Tzfira, T. (2007) Delivery of multiple transgenes to plant cells. Plant Physiol. 145, 1118-1128.

Doyon, Y., McCammon, J.M., Miller, J.C. et al. (2008) Heritable targeted gene disruption in zebrafish using designed zinc-finger nucleases. Nat. Biotechnol. 26, 702-708.

Durai, S., Mani, M., Kandavelou, K., Wu, J., Porteus, M.H. and Chandrasegaran, S. (2005) Zinc finger nucleases: customdesigned molecular scissors for genome engineering of plant and mammalian cells. Nucleic Acids Res. 33, 5978-5990.

Gallois, P. and Marinho, P. (1995) Leaf disk transformation using Agrobacterium tumefaciens-expression of heterologous genes in tobacco. Methods Mol. Biol. 49, 39-48.

Jasin, M., Moynahan, M.E. and Richardson, C. (1996) Targeted transgenesis. Proc. Natl Acad. Sci. USA, 93, 8804-8808.

Jefferson, R.A., Kavanagh, T.A. and Bevan, M.W. (1987) GUS fusions: beta-glucuronidase as a sensitive and versatile gene fusion marker in higher plants. EMBO J. 6, 3901-3907.

Kirik, A., Salomon, S. and Puchta, H. (2000) Species-specific doublestrand break repair and genome evolution in plants. EMBO J. 19, 5562-5566.

Lloyd, A., Plaisier, C.L., Carroll, D. and Drews, G.N. (2005) Targeted mutagenesis using zinc-finger nucleases in Arabidopsis. Proc. Natl Acad. Sci. USA, 102, 2232-2237.

Mandell, J.G. and Barbas, C.F. III (2006) Zinc Finger Tools: custom DNA-binding domains for transcription factors and nucleases. Nucleic Acids Res. 34, W516-W523.

Mani, M., Kandavelou, K., Dy, F.J., Durai, S. and Chandrasegaran, S. (2005) Design, engineering, and characterization of zinc finger nucleases. Biochem. Biophys. Res. Commun. 335, 447-457.

Meng, X., Noyes, M.B., Zhu, L.J., Lawson, N.D. and Wolfe, S.A. (2008) Targeted gene inactivation in zebrafish using engineered zinc-finger nucleases. Nat. Biotechnol. 26, 695-701.

Moehle, E.A., Rock, J.M., Lee, Y.L., Jouvenot, Y., Dekelver, R.C., Gregory, P.D., Urnov, F.D. and Holmes, M.C. (2007) Targeted gene addition into a specified location in the human genome using designed zinc finger nucleases. Proc. Natl Acad. Sci. USA, 104, 3055-3060.

Morton, J., Davis, M.W., Jorgensen, E.M. and Carroll, D. (2006) Induction and repair of zinc-finger nuclease-targeted doublestrand breaks in Caenorhabditis elegans somatic cells. Proc. Natl Acad. Sci. USA, 103, 16370-16375.

Paques, F. and Duchateau, P. (2007) Meganucleases and DNA double-strand break-induced recombination: perspectives for gene therapy. Curr. Gene Ther. 7, 49-66.

Porteus, M. (2008) Design and testing of zinc finger nucleases for use in mammalian cells. Methods Mol. Biol. 435, 47-61.

Porteus, M.H. and Baltimore, D. (2003) Chimeric nucleases stimulate gene targeting in human cells. Science, 300, 763.

Porteus, M.H. and Carroll, D. (2005) Gene targeting using zinc finger nucleases. Nat. Biotechnol. 23, 967-973.

Puchta, H. (2002) Gene replacement by homologous recombination in plants. Plant Mol. Biol. 48, 173-182.

Puchta, H., Dujon, B. and Hohn, B. (1993) Homologous recombination in plant cells is enhanced by in vivo induction of double strand breaks into DNA by a site-specific endonuclease. Nucleic Acids Res. 21, 5034-5040.

Puchta, H., Dujon, B. and Hohn, B. (1996) Two different but related mechanisms are used in plants for the repair of genomic double- strand breaks by homologous recombination. Proc. Natl Acad. Sci. USA, 93, 5055-5060.

Ramirez, C.L., Foley, J.E., Wright, D.A. et al. (2008) Unexpected failure rates for modular assembly of engineered zinc fingers. Nat. Methods, 5, 374-375.

Salomon, S. and Puchta, H. (1998) Capture of genomic and T-DNA sequences during double-strand break repair in somatic plant cells. EMBO J. 17, 6086-6095.

Sander, J.D., Zaback, P., Joung, J.K., Voytas, D.F. and Dobbs, D. (2007) Zinc Finger Targeter (ZiFiT): an engineered zinc finger/ target site design tool. Nucleic Acids Res. 35, W599-W605.

Santiago, Y., Chan, E., Liu, P.Q. et al. (2008) Targeted gene knockout in mammalian cells by using engineered zinc-finger nucleases. Proc. Natl Acad. Sci. USA, 105, 5809-5814.

Shaked, H., Melamed-Bessudo, C. and Levy, A.A. (2005) High frequency gene targeting in Arabidopsis plants expressing the yeast RAD54 gene. Proc. Natl Acad. Sci. USA, 102, 12265-12269.

Siebert, R. and Puchta, H. (2002) Efficient repair of genomic doublestrand breaks by homologous recombination between directly repeated sequences in the plant genome. Plant Cell, 14, 11211131.

Takahashi, T. and Komeda, Y. (1989) Characterization of two genes encoding small heat-shock proteins in Arabidopsis thaliana. Mol. Gen. Genet. 219, 365-372.

Terada, R., Urawa, H., Inagaki, Y., Tsugane, K. and lida, S. (2002) Efficient gene targeting by homologous recombination in rice. Nat. Biotechnol. 20, 1030-1034.

Tzfira, T. and White, C. (2005) Towards targeted mutagenesis and gene replacement in plants. Trends Biotechnol. 23, 567-569.

Tzfira, T., Frankmen, L., Vaidya, M. and Citovsky, V. (2003) Sitespecific integration of Agrobacterium tumefaciens T-DNA via double-stranded intermediates. Plant Physiol. 133, 1011-1023.

Tzfira, T., Li, J., Lacroix, B. and Citovsky, V. (2004) Agrobacterium T-DNA integration: molecules and models. Trends Genet. 20, 375-383.

Tzfira, T., Tian, G.-W., Lacroix, B., Vyas, S., Li, J., Leitner-Dagan, Y., Krichevsky, A., Taylor, T., Vainstein, A. and Citovsky, V. (2005) PSAT vectors: a modular series of plasmids for autofluorescent protein tagging and expression of multiple genes in plants. Plant Mol. Biol. 57, 503-516.

Urnov, F.D., Miller, J.C., Lee, Y.L., Beausejour, C.M., Rock, J.M., Augustus, S., Jamieson, A.C., Porteus, M.H., Gregory, P.D. and Holmes, M.C. (2005) Highly efficient endogenous human gene correction using designed zinc-finger nucleases. Nature, 435, 646-651.

Van der Hoorn, R.A., Laurent, F., Roth, R. and De Wit, P.J. (2000) Agroinfiltration is a versatile tool that facilitates comparative analyses of Avr/cf-9-induced and Avr4/cf-4-induced recrosis. Mol. Plant Microbe Interact. 13, 439-446.

Wright, D.A., Townsend, J.A., Winfrey, R.J. Jr, Irwin, P.A., Rajagopal, J., Lonosky, P.M., Hall, B.D., Jondle, M.D. and Voytas, D.F. (2005) High-frequency homologous recombination in plants mediated by zinc-finger nucleases. Plant J. 44, 693-705.

Wright, D.A., Thibodeau-Beganny, S., Sander, J.D. et al. (2006) Standardized reagents and protocols for engineering zinc finger nucleases by modular assembly. Nat. Protoc. 1, 1637-1652.

Zeevi, V., Tovkach, A. and Tzfira, T. (2008) Increasing cloning possibilities using artificial zinc finger nucleases. Proc. Natl Acad. Sci. USA, 105, 12780-12790.

Zhang, X., Henriques, R., Lin, S.S., Niu, Q.W. and Chua, N.H. (2006) Agrobacterium-mediated transformation of Arabidopsis thaliana using the floral dip method. Nat. Protoc. 1, 641-646. 